\title{
HISTOLOGICAL STUDY ON THE EFFECT OF THE IMMUNOSUPPRESSIVE DRUGS ON THE MUCOUS MEMBRANE AND SALIVARY GLANDS OF THE ALBINO RATS TONGUE
}

\section{Loaa Mahmoud ${ }^{1}$, Laila Ghali ${ }^{2}$, Manar Selim ${ }^{3}$}

DOI: $10.21608 / d s u .2021 .18795 .1013$

Manuscript ID: DSU-1910-1013 (R7)

\section{KEYWORDS}

Immunosuppressive agents,

Organ transplantation, Rats,

Tongue \& Tacrolimus.

- E-mail address:

Dr.loaadiaa1984@gmail.com

1. Demonstrator in Oral Biology Department, Faculty of Dentistry, Future University

2. Professor of Oral Biology, Faculty of Oral and Dental Medicine, Cairo University

3. Associate professor of Oral biology, Faculty of Dentistry, Suez Canal University.

\begin{abstract}
Introduction: Tacrolimus is a macrolide calcineurin inhibitor which has been shown to decrease acute and chronic rejection, improved renal function over the longterm post-transplant. Tacrolimus is considered the drug of choice in immunosuppressive therapy for chronic diseases irresponsive to corticosteroids, autoimmune diseases and organ transplantation system. Aim: The aim of the investigation was to study the effect of the immunosuppressive drug Tacrolimus on the lingual mucous membrane and salivary glands of treated rats through hematoxylin and eosin stained sections to detect any microscopic changes in the structure of the mucous membrane and salivary glands of the tongues \& Masson's trichrome stained sections for detection of the integrity of collagen. Material and Methods: Thirty healthy adult male albino rats with body weight ranging from 150 to $180 \mathrm{gm}$ were used in this investigation. The animals were divided into two groups 15 animals each. Group 1: served as control, group 2: animals were treated with the immunosuppressive drug tacrolimus dissolved in distilled water in a daily oral dose of $0.5 \mathrm{mg} / \mathrm{kg}$ body weight. At the end of the experiment the rats of different groups were euthanized by cervical dislocation. The tongues were dissected out, fixed, processed, embedded in paraffin, sectioned and stained with hematoxylin and eosin for histological evaluation \& Masson's trichrome stain for demonstration of collagen fibers. Results: The light microscopic examination of group I showed the normal histological features of both surface epithelium and underlying lamina propria, while group II Treated with tacrolimus revealed atrophic and degenerative changes that involved the surface epithelium and lamina propria of both the dorsal and ventral surfaces of the tongue as well as the lingual salivary glands. Conclusion: Tacrolimus administration showed structural alterations in the lingual mucous membrane and the lingual salivary glands which will certainly affect the taste sensation.
\end{abstract}

\section{INTRODUCTION}

Organ transplantation has become a viable treatment for an increasing number of patients suffering from irreversible organ failure. There are different types of transplants including autografts, allografts, isografts and xenografts which is extremely dangerous ${ }^{(1)}$.

Medical categories of rejection include hyperacute, acute and chronic rejection ${ }^{(2)}$. Hyperacute rejection manifests within minutes 
after transplant and if tissue is left implanted it brings systemic inflammatory response of high risk. Acute rejection occurs in all transplants unless immunosuppression is achieved while chronic rejection means loss of function via fibrosis leading to loss of graft function ${ }^{(3)}$. Immunosuppressive agents are a class of drugs reducing the strength of the body's immune system. Steroids, anti-proliferative agents, calcineurin inhibitors and target of rapamycin inhibitors (TOR) are different types of immunosuppressive agents ${ }^{(4)}$.

Tacrolimus (macrolide calcineurin inhibitors) has been shown to decrease acute and chronic rejection, improved renal function over the longterm post-transplant with lower risk factors. Tacrolimus is available as intravenous injection, oral administration or topical ointment.

Tacrolimus has been used to suppress the inflammation associated with ulcerative colitis irresponsive to high doses of systemic corticosteroids with better side effects ${ }^{(5)}$. Tacrolimus is used as dermatological treatment in atopic dermatitis, erosive mucosal lichen planus and Crohn's disease of the mouth ${ }^{(6)}$.

The cancer risk of immunosuppressive drugs is a major concern as the incidence of malignancy is significantly higher for organ transplant recipients than for general population.

The FDA advised weighing up the risks of treatment against the benefits and they also indicated that patients should not use these agents if their immune system was already compromised ${ }^{(7)}$. Tacrolimus is considered the corner stone in immunosuppressive therapy for chronic diseases irresponsive to corticosteroids, autoimmune diseases and organ transplantation system ${ }^{(8)}$.
Mechanism of action of tacrolimus; Tacrolimus is a macrolide calcineurin inhibitor. In T-cells, activation of the T-cell receptor normally increases intracellular calcium, which acts via calmodulin to activate calcineurin. Calcineurin then dephosphorylates the transcription factor nuclear factor of activated T-cells (NF-AT), which increase the activity of gene coding for IL-2 and related cytokines. Tacrolimus prevents the dephosphorylation of NF-AT ${ }^{(9)}$.

Tacrolimus has a wide range of drug-drug and food-drug interactions. Because of its variable pharmacokinetics and narrow therapeutic index so monitoring drug concentrations is essential to avoid the risk of over and under immunosuppression ${ }^{(10)}$.

Side effects include infection, cardiac damage, hypertension, liver and kidney problems (tacrolimus nephrotoxicity) ${ }^{(11)}$, hyperkalemia, hypomagnesemia, diabetes mellitus, lung damage and neuropsychiatric problems such as loss of appetite, insomnia, confusion, weakness, depression, cramps, seizures, tremors and catatonia ${ }^{(12-13)}$.

Infections have been reported to occur in $\leq 50 \%$ of patients treated with tacrolimus. It may increase the severity of existing fungal or viral conditions such as herpes zoster ${ }^{(14)}$

It's contraindicated to use tacrolimus in case of pregnancy, breast feeding and for infants (15). Ultra-violet light exposure and grapefruit juice administration could be critical with tacrolimus use. Precautions involve oliguria, occlusive dressing and neoplastic diseases ${ }^{(16)}$.

The aim of the present investigation is to study the effect of the immunosuppressive drug Tacrolimus on the lingual mucous membrane and salivary glands of albino rats. 


\section{MATERIAL AND METHODS}

Thirty healthy adult male albino rats with body weight ranging from 150 to $180 \mathrm{gm}$ were used in this investigation. The animals were divided into two groups 15 animals each. They were supplied standard natural diet and drinking tap water adlibitum. The animals were divided as follows:-

Group 1: served as control and received distilled water using curved metallic oropharyngeal tube.

Group 2: animals were treated with the immunosuppressive drug tacrolimus dissolved in distilled water in a daily oral dose of $0.5 \mathrm{mg} / \mathrm{kg}$ body weight using a curved metallic oropharyngeal tube. Tacrolimus is supplied in tablet form that were ground to be easily dissolved in distilled water in a concentration of $0.15 \mathrm{mg} / \mathrm{ml}$. The daily dose for a rat weighing $150 \mathrm{gm}$ was $0.5 \mathrm{ml}$.

The percentage of mortality for each group was calculated and recorded. Gross examination of the head and neck of each animal was carried out throughout the whole experimental period which lasted for three months.

At the end of the experiment the rats of different groups were euthanized by cervical dislocation under general anesthesia. The tongues were dissected, fixed in 10\% natural buffered formalin, processed, embedded in paraffin, sectioned and stained with:-

- Hematoxylin and eosin for histological evaluation.

- Masson's trichrome stain for demonstration of collagen. The collagen density was evaluated by using image $\mathrm{J}$ software and the mean value was calculated and statistically analyzed.

\section{RESULTS}

Mortality rate: tacrolimus resulted in $10 \%$ mortality in rats of group II.

Clinical findings: a constant finding was the presence of a film of white coat on the dorsal surface of the tongue resembling fungal infection of candida albicans.

\section{Histological Results:-}

\section{A- Haematoxylin and Eosin stained sections:-}

\section{Group I animals (control group):-}

It showed the normal histological features of both surface epithelium and underlying lamina propria. The mucous membrane of the dorsal surface of the tongue was formed of stratified squamous epithelium with normal protruding filliform and the fungiform papillae while the circumvallate papillae were not protruding from the surface. The underlying lamina propria was well vascularized, formed of dense bundles of collagen fibers with connective tissue cells.

There were normal pure serous acini situated below the circumvallate papillae forming the Von Ebner salivary glands. Pure mucous salivary glands (Weber glands) were formed of normal mucous acini (Fig. 1).

\section{Group II animals (Treated with Tacrolimus):-}

Examination revealed atrophic and degenerative changes that involved the surface epithelium and lamina propria of both the dorsal and ventral surfaces of the tongue as well as the lingual salivary glands (Fig. 2). 


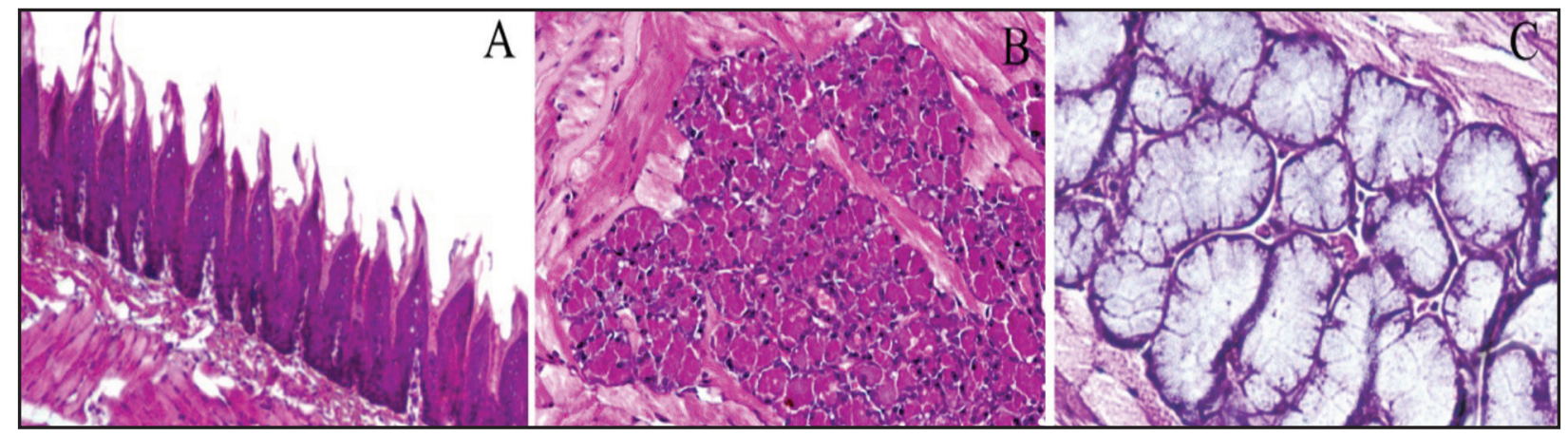

Fig. (1) A photomicrograph of the dorsal surface of the tongue of group I animals showing: A normal filiform papillae and lamina propria with collagen fibers (H\&E, orig.mag.200). B: The pure serous acini of Von Ebner salivary glands (H\&E, orig. mag. 200). C: The pure mucous acini of weber salivary gland (H\&E, orig. mag. 400).

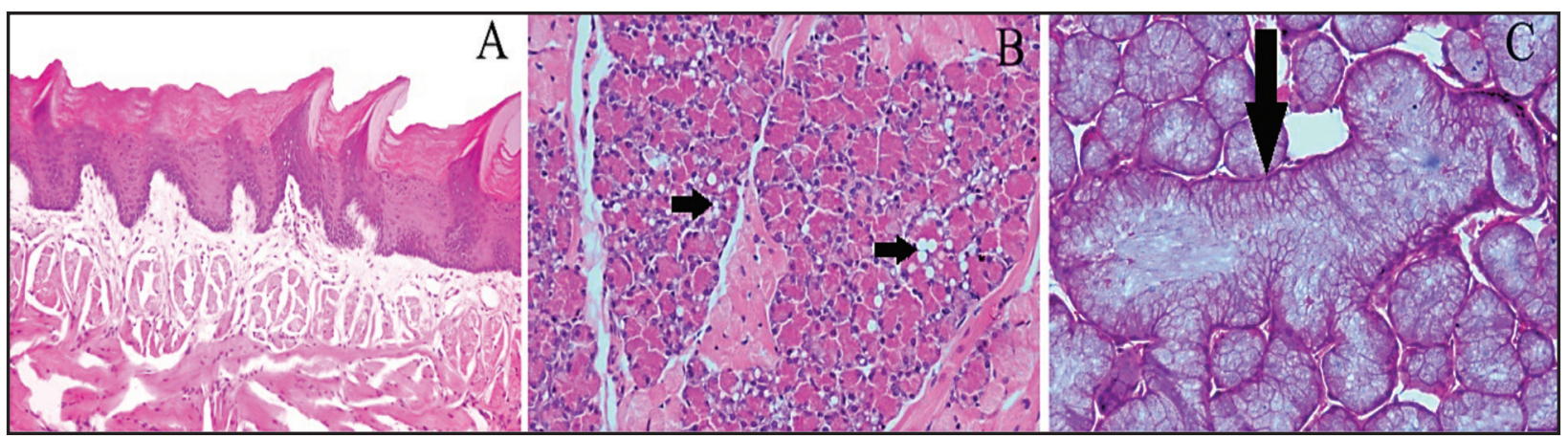

Fig. (2) A photomicrograph of the dorsal surface of the tongue of group II animals showing: A: Atrophy and decrease in the length and number of filiform papillae (H\&E orig.mag.200). B: Degenerating serous acinar cells with a lot of intracytoplasmic vacuolization (black arrows) (H\&E, orig.mag.200). C: Degenerating mucous acini with cystic transformation and degeneration of collagen fibers (black arrow) (H\&E, orig.400).

\section{B - Masson's trichrome stain:}

The histological sections of tongue of group I animals stained with Masson's trichrome stain revealed a strongly positive staining of the collagen fibers of the lamina propria (Fig. 3A), while group II animals (immunosuppressive treated group) revealed weakly to moderately positive staining reaction (Fig. 3B), indicating degeneration of the collagen fibers, these results were statistically confirmed, there was significant difference between the optical density of the Masson's trichrome stain between the two groups, as $\mathrm{P}$-value $<0.001$ (Table.1)

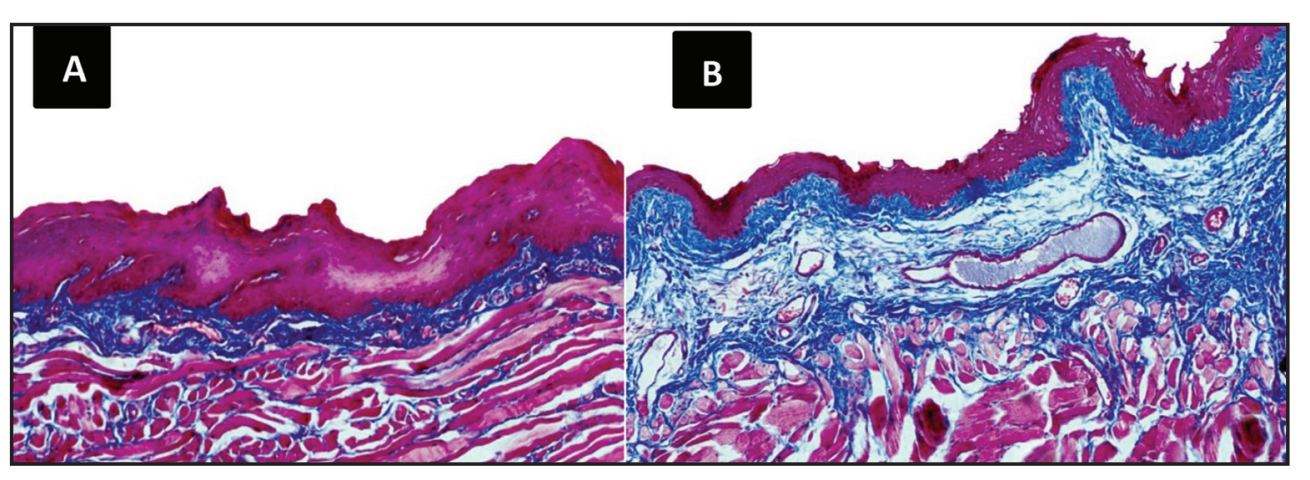

Fig. 3: A photomicrograph of the ventral surface of the tongue showing: A) group I animals showing strongly positive staining reaction of the collagen fibers of lamina propria to masson's trichrome stain (orig.mag.200). B) group II animals showing moderate positive reaction of the collagen fibers of lamina propria to Masson's trichrome stain (orig.mag.100). 
Table (1) Masson's trichrome stain results of the control group and immunosuppressive treated group.

\begin{tabular}{ccc}
\hline Group & Mean ${ }^{ \pm}$Std. Deviation & P value \\
\cline { 1 - 2 } Control & $130.5^{ \pm} 14.9$ & $*<0.001$ \\
\cline { 1 - 2 } Treated & $66.0^{ \pm 10.6}$ & \\
\hline
\end{tabular}

*A significant $P$ value was found between treated and control groups regarding the mean $(P$-value $<0.001)$

\section{DISCUSSION}

The role of the immune system is to defend the body of vertebrates against infection. There are two classes of lymphocytes, B lymphocytes which make antibodies and $\mathrm{T}$ lymphocytes which are responsible for cell mediated immune response ${ }^{(17)}$. The goal of immunosuppression is to control graft rejection. After 1962, the protocols for organ transplantation in humans called for weakening of the recipient's immune system by immunosuppressive drugs after rather than before transplantation ${ }^{(18)}$.

The histological results of the present study revealed that tacrolimus produced variable degrees of atrophic changes in the epithelium and the different types of tongue papillae. There was loss of the normal shape of each tongue papilla and degeneration of the cells of taste buds inside it.

The epithelium of the basal and spinous cell layers showed cytoplasmic vacuolization. Also the epithelial cells of the serous and mucous acini showed marked vacuolization.

Collagen fibers in the subepithelial lamina propria of the tongues suffered dissociation were seen in the group treated with tacrolimus. Masson's trichrome stained sections confirmed the marked degeneration and dissociation of the collagen fibers in the tissues under investigation.
Helenius ${ }^{(19)}$, studied the orthopedic complications after solid organ transplantation in childhood. He reported that immunosuppressive drugs, can compromise the quality of bone in children who have undergone solid organ transplantation. Fractures, scoliosis, and joint and spinal degeneration are common in patients who received solid organ grafts as children. The effect of immunosuppression on the metabolism of cartilage cells, structure of the cartilage matrix and changes in collagen metabolism were clear.

The intracytoplasmic vacuolization present in the serous acini of Von Ebner and the cystic transformation of mucous acini of salivary glands present in tongues of tacrolimus treated rats are possibly due to uncontrolled infections. The incidence of infection in transplant patients is very high. Almost $80 \%$ of all transplant patients had infection and $40 \%$ of deaths among transplant patients are due to complications of infections. The incidence of infections differs according to type, intensity and duration of immunosuppressive therapy ${ }^{(20)}$. It was reported that after 60-day treatment with any of the immunosuppressants both parotid and submandibular glands showed histomorphological alterations involving the parenchymal elements and connective tissue septa ${ }^{(21)}$.

It has been stated that in patients receiving intravenous tacrolimus as "rescue" therapy for refractory renal or hepatic allograft rejection, initial nephrotoxicity is seen in nearly all patients with occurrence of epithelial vacuolization in clinical biopsy material which suggests a direct toxic effect of tacrolimus on the renal tubule. The principal associated morphologic finding was arteriolar myocyte vacuolization, which may reflect a direct toxic effect of the drug on smooth-muscle cells. This direct toxicity is morphologically characterized by tubular degeneration and atrophy ${ }^{(22)}$. These findings 
support the results in the present investigation of tacrolimus effect on rat tongue as vacuolization was clear in the epithelium of the mucous membrane and in the acini of salivary glands.

Similar to the present findings structural alterations in the seminiferous tubules of rats treated with the immunosuppressor tacrolimus were reported. The seminiferous tubules showed disarranged epithelial layers and lumen filled with detached germ cells; lack of germ cells were evident in the atrophied tubules. In the altered tubules, the sertoli cell nuclei exhibited irregular shape. Adjacent to these altered nuclei, vacuolar spaces were often observed. A significant reduction in the tubular and epithelial areas was observed in the testes of rats treated with Tacrolimus ${ }^{(23)}$.

According to the previous opinions and investigations, the use of topical and systemic tacrolimus, after weighing the possible risks and benefits, could be helpful in immunosuppression as it controls graft rejection successfully with decreasing death rate. However, its use should be in limits and under supervision due to its high toxicity and side effects.

\section{CONCLUSION}

Tacrolimus administration showed structural alterations in the lingual mucous membrane and the lingual salivary glands which will certainly affect the taste sensation.

\section{REFERENCES}

1. Jean-Baptiste MI, Patrick BR, Catherine GU, Didier PL. Cell-free arterial grafts: Morphologic characteristics of aortic isografts, allografts, and xenografts in rats. J Vasc Surg 1994; 19: 446-456.

2. Ingulli EL. Mechanism of cellular rejection in transplantation. Ped Nephrol 2010; 25: 61-74.
3. Racusen LC, Solez KI, Colvin RO. Fibrosis and atrophy in the renal allograft: interim report and new directions. Am J Trans 2002; 2: 203- 206.

4. Anna LT, Christopher JE, Andrew JB. Immunosuppressive agents in solid organ transplantation: Mechanisms of action and therapeutic. Crit Rev Onco/Hema 2005; 56:23-46.

5. Yamamoto S, Nakase H, Mikami S, Takeda Y. Long-term effect of tacrolimus therapy in patients with refractory ulcerative colitis. Alim Pharma \& Therap 2008; 28:589-597.

6. Nakagawa H, Tishibashi Y. Tacrolimus ointment for topical dermatitis. Lancet 1994; $344: 883$.

7. Ormerod AD. Topical tacrolimus and pimecrolimus and the risk of cancer: how much cause for concern? Brit J Derma 2005; 153:701-705.

8. First M. Tacrolimus based immunosuppression. J Nephrol 2004; 17: 25-30.

9. Ganong WF. Review of Medical Physiology $2^{\text {nd }}$ edition McGraw Hill. Companies Inc., New York. 2003; 518.

10. Jusko WJ, Thomson AW, Paul MC. Consensus document: therapeutic monitoring of tacrolimus (FK506). Ther Drug Monit 1995; 17: 606-614.

11. Naesens MA, Kuypers RJ, Sarwal MI. Calcineurin inhibitor nephrotoxicity. Clin J Am Soc Nephrol 2009; 4 :481-509.

12. Miwa YU, Isozaki TA, Wakabayashi KU. Tacrolimusinduced lung injury in a rheumatoid arthritis patient with interstitial pneumonitis. Mod Rheumatol 2008; 18 : 208-211.

13. O’Donnell MM, Williams JP, Deysenko LE. Catatonic mutism after liver transplant rapidly reversed with larazepam. Gen Hosp Psych 2007; 29: 280-281.

14. Simon MG, Ingvar BJ, Zahra TO, David BA. The effect of tacrolimus (FK506) on intestinal barrier function and cellular energy production in humans. Gastroenterol 1998; $115: 67-74$.

15. Moxey MM, Kay C, Light JA. Increased incidence of insulin-dependent diabetes mellitus in pediatric renal transplant patients receiving tacrolimus (FK506). Transplant 1998; 65: 617-619.

16. Hirobumi KO, Toru AB, Hiroshi HA, Sachiko SU. Efficacy and safety of tacrolimus (FK506) in treatment of rheumatoid arthritis: a randomized, double blind, placebo controlled dose-finding study. J Rheumatol 2004; 31: 243-251. 
17. Kurosaki TO, Kometani KO, Ise WA. Memory B cells. Nat Rev Immunol 2015; 15: 149-159.

18. Starzl TE. Immunosuppressive therapy and tolerance of organ allografts. New Eng J Med 2008; 358 : 407-411.

19. Ilkka HE, Hannu JA, Ville RE, Sari SA, Christer HO. Therapy Insight: orthopedic complications after solid organ transplantation in childhood. Nat Clin Prac Nephrol 2007; 3: 96-105.

20. Razonable RR, Limaye AP. Cytomegalovirus infection after solid organ transplantation. Transplant Infections. Fourth edition. Switzerland: Springer, Cham 2016:441-475.
21. Luís CS, Bruno SH, Leila SC, Cleverton RD, Marcelo NM. The long-term administration of calcineurin inhibitors decreases antioxidant enzyme activity in the rat parotid and submandibular salivary glands. Life Sci 2015; 134:1-8.

22. Parmjeet SR, Thomas ES, Anthony JD. Tacrolimus (FK506)-Associated Renal Pathology. Adv Anat Pathol 1997; 4 : 265-276.

23. Breno HC, Paulo SC, Luís CS, Sandra MM, Estela SC. Structural alterations in the seminiferous tubules of rats treated with immunosuppressor tacrolimus. Reprod Biol Endocrinol 2009; 7 : 7-19. 